\title{
Global-local finite element analysis of riveted railway bridge connections for fatigue evaluation
}

\author{
B.M. Imam, T.D. Righiniotis \& M.K. Chryssanthopoulos \\ Faculty of Engineering and Physical Sciences, University of Surrey, UK
}

\begin{abstract}
Recent studies have found that stringer-to-cross-girder connections in riveted railway bridges are susceptible to fatigue cracking. Fatigue damage in these connections is caused by secondary, deformation induced effects. These effects are difficult to interpret in terms of a single, applied stress descriptor, which is customarily used in an S-N assessment. In order to address this problem, and, following the work published recently by the authors (Imam et al. 2006, 2007), in this paper the results of a global-local finite element analysis of a riveted railway bridge are used within the context of the theory of critical distances (TCD). Here, using the TCD in the way proposed by Taylor (Bellett et al. 2005), fatigue damage is shown to converge upon mesh refinement. Comparisons of the TCD-based method with its more traditional, detail-specific S-N counterpart, reveal that the latter can underestimate fatigue damage in some cases by a factor of 3.5 .
\end{abstract}

\section{INTRODUCTION}

In the past few years, fatigue of riveted railway bridges connections has received considerable attention. A number of damaged cases have been reported for stringer-to-cross-girder connections both in railway and highway bridges confirming that these details are susceptible to fatigue cracking (Al-Emrani 2002). Fatigue damage in these connections is caused by deformation induced secondary stresses which were not taken into account during the design of these bridges. This is because these secondary effects are, even today, often very difficult to analyse, requiring sophisticated models. In this respect, the best way of avoiding this type of fatigue damage in new bridges is good detailing. For existing bridges however, it is still a challenge to find practical, yet accurate, models to assess the remaining life of these details.

All modern fatigue related codes of practice employ the S-N method for the fatigue assessment of bridge details (BS 5400 1980, AASHTO 1998). A specific class, which depends on geometry and loading, is assigned to the bridge detail in question. The fatigue life of the detail is then estimated by using its code-specified S-N curve with the nominal stress applied to it. This method can be easily used in conjunction with tensile riveted details such as bottom flange-toweb connections in built-up beams, cover plates and gusset connections between the primary members of a bridge with hangers, diagonals and bracings. In such details, it is straightforward to identify the nominal stress to be used for fatigue calculations and, owing to a large number of fatigue tests carried out on such details, their S-N curves are well defined.

On the other hand, it is almost impossible to identify the nominal stress in stringer-to-crossgirder connections and this is due to their complex behaviour which involves secondary effects and depends on the interaction of different components such as rivets, angle clips and parts of the primary bridge members such as flanges and webs. Although few full-scale fatigue tests have been carried out in such connections (Abouelmaaty et al. 1999, Al-Emrani 2005), the test results were not expressed in a meaningful way on an S-N basis due to the above mentioned difficulties. 
These difficulties can be overcome by focusing on the local stresses in the vicinity of the stress concentrations, which explicitly take into account their effect, rather than having to identify a nominal stress value and use it with code-specified S-N curves which implicitly account for stress concentration effects. Local stress distributions can nowadays be obtained with precision by using the finite element (FE) method even for the most complex details and geometries. However, the maximum stress obtained at the point of stress concentration (notch tip) and the stress distribution around its vicinity is sensitive to the mesh size used in the FE model. Overconservative predictions are obtained when fatigue damage is estimated using this maximum stress. During recent years, it has been proposed that rather than considering the maximum stress only, the entire stress distribution ahead of the stress concentration should be taken into account for fatigue damage calculations (Bellett et al. 2005). This theory, called the Theory of Critical Distances (TCD), was developed by Taylor and will be presented in more detail later in the paper.

In the past, the authors have employed the $\mathrm{S}-\mathrm{N}$ method for the estimation of the fatigue damage of stringer-to-cross-girder connections (Imam et al. 2006). Through this global investigation, which was carried out by using a crude FE model consisting exclusively of shell elements, all the connections in a typical, short-span, riveted railway bridge were ranked according to their estimated fatigue damage and, therefore, the most critical one identified. The authors have subsequently refined their global model in the region of the most critical connection using submodelling techniques and three-dimensional brick elements (Imam et al. 2007), as shown in Figure 1. The exact geometrical features and all the connection components such as rivets, angle clips and parts of the stringer and cross-girder webs were modelled in detail. Other features pertinent to riveted connections such as the rivet clamping force and the friction between the connected parts were also explicitly accounted for. The results obtained from this global-local analysis were presented in a qualitative context since notch tip stresses were considered.

In this paper, the TCD is used in order to obtain fatigue damage estimates through the globallocal model and compare them with the estimates obtained in the past through the global model and the S-N method (Imam et al. 2006). For this reason, the BS 5400 (1980) medium traffic trains are initially traversed over the bridge. Histories of the maximum principal stress are obtained at various critical locations within the connection. By using the TCD in conjunction with the plain (un-notched) material S-N curve and Miner's rule, the different components of the connection are ranked according to their estimated fatigue damage. Therefore, the most critical hot-spots of the connection where fatigue cracking is most likely to occur are identified. The damage estimates obtained from this more refined model are compared to its global model counterpart. The effect of rivet clamping force on fatigue damage is also quantified.

\section{THE THEORY OF CRITICAL DISTANCES (TCD)}

According to the TCD, all the physical processes leading to crack initiation are assumed to take place within a finite area/volume ahead of the stress concentration, called the process zone. Therefore, the fatigue behaviour depends on the elastic stress distribution within that region and not on a single stress concentration at a single point, as postulated by the traditional theory. The size of the process zone can be identified using two material properties: the plain specimen fatigue limit $\Delta \sigma_{0}$ and the crack propagation threshold $\Delta K_{t h}$. Within the context of the TCD, a critical distance, which characterises the region within which stresses are to be considered, is defined as (Taylor 1999):

$$
L=\frac{1}{\pi}\left(\frac{\Delta \mathrm{K}_{t h}}{\Delta \sigma_{0}}\right)^{2}
$$

It has to be noted that, since $\Delta K_{t h}$ and $\Delta \sigma_{0}$ are both material properties, Equation 1 implies that $L$ will also be a material property.

There are several ways of implementing the TCD, depending on the stresses considered for further calculations. The simplest ways are by considering the maximum principal stress at a single point (point method), a distance of $L / 2$ from the notch tip, or by averaging the maximum principal stress along a distance of $2 L$ (line method) (Bellett et al. 2005).Two additional options 
of the TCD is to average the maximum principal stress over a semi-circular area (area method) or over a hemisphere (volume method) (Bellett et al. 2005), both centred at the tip of the notch and having a radius of $1.32 \mathrm{~L}$ and $1.54 \mathrm{~L}$ respectively.

Having established the 'characteristic' stress by using one of the previously mentioned methods, this is then compared with the Constant Amplitude Fatigue Limit (CAFL) of the plain (unnotched) material in order to determine whether fatigue failure will occur. Comparison of experimental and analytical results on a wide range of notch geometries (circular holes, fillets etc.) and materials (steel, cast iron, aluminum) showed that the TCD is capable of predicting fatigue strength within an error margin of 20\% (Taylor \& Wang 2000, Susmel \& Taylor 2003).

The TCD has been primarily employed to determine the CAFL of all types of stress concentrations but it has also been verified being capable of estimating fatigue life under variable amplitude loading as well (Taylor et al. 2000). This was achieved by using the characteristic stress in conjunction with Miner's rule and the plain material S-N curve. Furthermore, the accuracy of the TCD for the fatigue assessment of welded joints (Taylor et al. 2002), under multiaxial stress conditions (Susmel 2004) and for fretting fatigue problems (Araujo et al. 2007) has also been verified.

Following this brief discussion on the TCD, the FE analysis is presented in the next section. In this investigation, the TCD is used in conjunction with variable amplitude loading resulting from the passage of trains over a short-span, riveted bridge in order to estimate the fatigue damage and the remaining life of a critical connection. Due to the three-dimensional nature of the notches in the connection, the volume method of the TCD is employed.

\section{FINITE ELEMENT ANALYSIS}

\subsection{Bridge finite element model}

The global-local FE model of the bridge, which is deemed to be representative of a large number of short-span, riveted railway bridges in the UK and across Europe, is shown in Figure 1a. Figure 1b depicts in detail the connection shown in Figure 1a as well as the nomenclature used to identify its components. The entire model, which was described in full detail and validated in previous studies (Imam et al. 2007), consists of approximately 67000 brick elements and 20000 shell elements. The FE analyses are carried out assuming linear elastic material behaviour. Two values of clamping stress are assumed for all rivets, i.e. a low to moderate clamping stress of $100 \mathrm{MPa}$ and a high clamping stress of $200 \mathrm{MPa}$.

The loading over the bridge consists of the BS 5400 (1980) medium traffic trains. These are traversed in $1 \mathrm{~m}$ steps over one track of the bridge, as shown in Figure 1a, up to the point of load repetition caused by the passage of similar wagons following the engine car. The train axle loads are applied quasi-statically directly on the top flange of the stringers thus neglecting dynamic amplification and any beneficial effects associated with the load spread on the rails and sleepers.
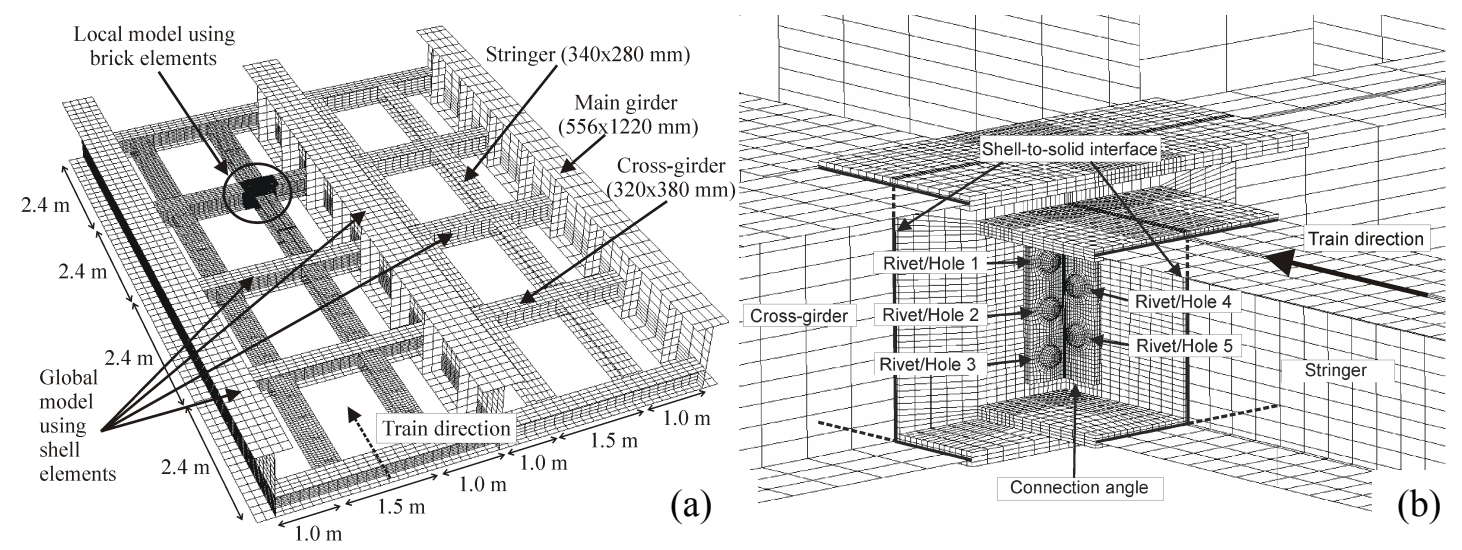

Figure 1. (a) Finite element model of the riveted bridge and (b) Close-up view of the connection and hole/rivet nomenclature. 


\subsection{Material properties}

The bridge is assumed to be constructed using wrought iron, a material that was used, rather extensively, for this purpose towards the latter part of the $19^{\text {th }}$ century. In the UK alone, it is estimated that there are around 6000 wrought iron bridges, similar to the one shown in Figure 1a. Experimental studies have shown that the value of Young's modulus for wrought iron varies between 170 and $223 \mathrm{GPa}$ (Cullimore 1967, Bruhwiler et al. 1990, Moy et al. 2004), having an average value of approximately $200 \mathrm{GPa}$. This value is used here as well as a Poisson's ratio of 0.3 .

With regard to the crack propagation threshold, a value of $\Delta K_{t h}=13.5 \mathrm{MPa} \cdot \mathrm{m}^{1 / 2}$ is assumed. This value was suggested by Helmerich et al. (2007) following a series of crack growth experiments on wrought iron at a load ratio of 0.1 . For the fatigue limit $\Delta \sigma_{0}$ of plain wrought iron material, the value of $183 \mathrm{MPa}$ is used, which is based on fatigue tests at a load ration of 0.1 (Cullimore 1967).

\subsection{Fatigue damage calculation}

The critical regions within the connection were identified in a previous study by the authors (Imam et al. 2007). These were typically located along the perimeter of the rivet holes on the face of the connection angle in contact with the cross-girder and stringer webs, along the angle fillet, and around the perimeter of the rivets at the head-to-shank intersection. The fatigue damage and the remaining fatigue life of the connection are estimated in this investigation.

For this purpose, the critical volumes to be used within the context of the TCD are first determined. These are shown in Figure 2 for the different critical regions within the connection, where the dotted lines indicate the boundary of the critical volume, whose characteristic dimension is $1.54 \mathrm{~L}$. The brick elements considered for the estimation of the characteristic stress to be used for fatigue damage calculations are shown as shaded in Figure 2. Partially included, within the critical volume, elements are here arbitrarily considered for further calculations when at least half of their volume lies within the critical volume. The maximum principal stresses are obtained at the centroids of these elements since these represent an average, within the element, value. The characteristic stress of interest, $\sigma_{\text {ave }}$, to be used for fatigue damage calculations, is then calculated by averaging, over all the elements, the centroidal stresses.
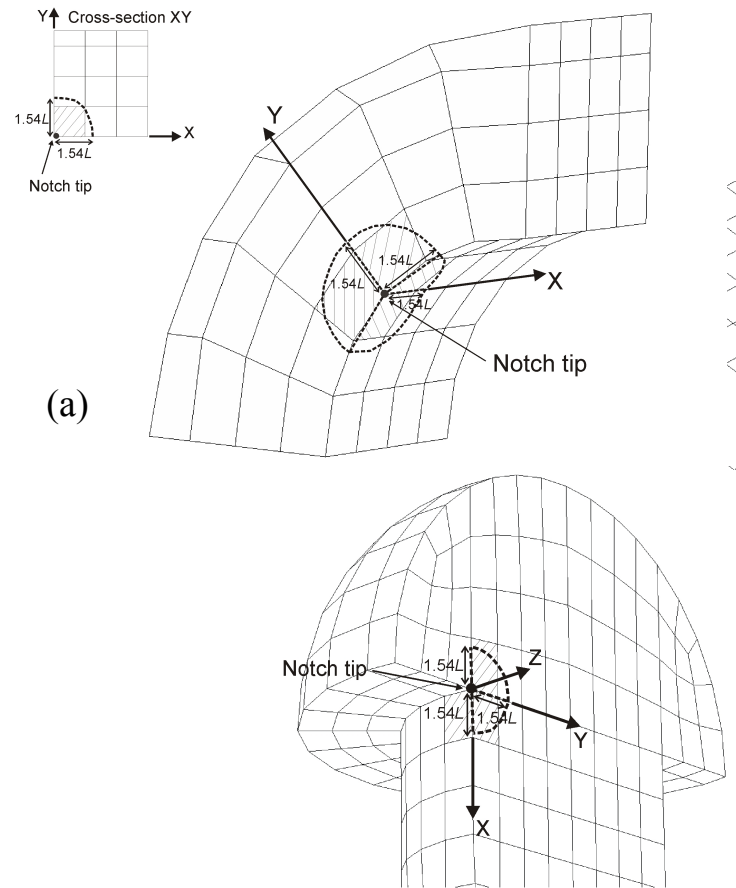

(b)

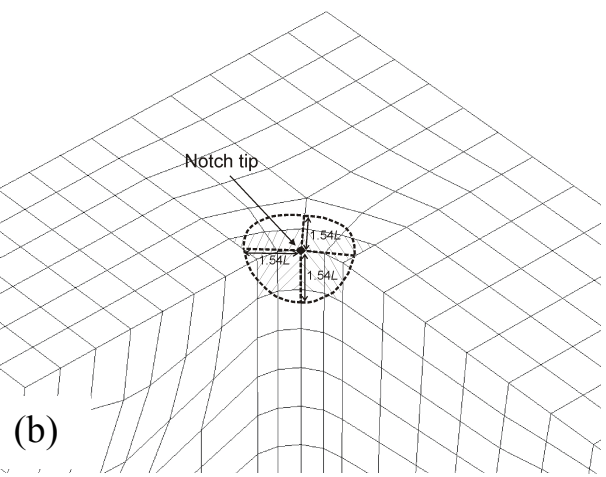

(c)

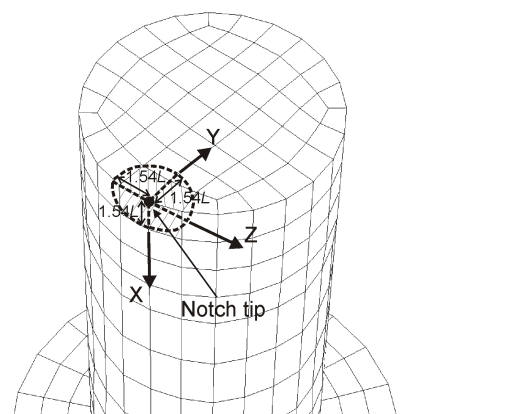

Figure 2. (a) Critical volume around the hole perimeter; (b) Critical volume around the angle fillet;

(c) Critical volume around the rivet perimeter. 
It has to be mentioned that at each of the locations shown in Figure 2, there exist several critical volumes associated with different notch tips. For example, considering the holes (see Fig. 2a), the above procedure is carried out, at each analysis step, for all the notch tips all around the circumference of the hole. Several stress histories are thus generated corresponding to the different notch tips. Each of these stress histories is then converted into stress range $\left(\Delta \sigma_{\text {ave }}\right)$ blocks and Miner's rule is applied on the plain material S-N curve in order to calculate the fatigue damage. By comparing the damage estimated for the different notch tips at each location, the most damaging tip is then identified. For the purposes of this study, a single-slope S-N curve (slope 1/5) with a fatigue limit of $\Delta \sigma_{0}=183 \mathrm{MPa}$, defined at $N_{F L}=2 \times 10^{6}$ (Cullimore 1967), is used for damage calculations. Since train loading is of variable amplitude loading, the CAFL is disregarded and the S-N curve is extended below $\Delta \sigma_{0}$. Thus, the fatigue damage for a single train passage, which produces $k$ stress blocks, will be given as

$$
D_{l 1}=\frac{1}{N_{F L}\left(\Delta \sigma_{0}\right)^{5}} \sum_{1}^{k} n_{i}\left[\left(\Delta \sigma_{\text {ave }}\right)_{i}\right]^{5}
$$

where $\left(\Delta \sigma_{\text {ave }}\right)_{i}=i^{\text {th }}$ stress range; $n_{i}=$ corresponding number of cycles.

By multiplying the damage values obtained from the single passage of each train over the bridge (Equation 2) by their corresponding frequencies given in BS 5400 (1980) and summing up over the years, the total fatigue damage of the different locations within the connection can be calculated. The accumulation of damage is assumed to initiate at the year 1970 with the introduction of the BS 5400 medium traffic. Justification for this is provided from previous investigations by the authors which showed that the damage that has accumulated in the connections of this type of bridges by the passage of historical train traffic is insignificant (Imam et al. 2006).

\section{RESULTS AND DISCUSSION}

\subsection{Convergence study}

A common difficulty encountered during the fatigue assessment of stress concentrations through FE analysis is that of mesh convergence. Use of the traditional theory, which relies on stress definition at a single point, i.e. the notch tip, often does not lead to convergence resulting in a continuous increase in the notch tip stress with mesh refinement. As a result, fatigue damage becomes unbounded.

Within the context of the TCD, which relies on the entire stress distribution ahead of the notch tip, a convergence study was carried out in order to examine the effect of mesh refinement on fatigue damage. Three different mesh densities were investigated parts of which are shown in Figure 3. Differences between mesh 1 and mesh 2 are brought about by increasing the number of elements along the thickness and along the depth of the angle fillet. For mesh 3 , the number of elements along the thickness direction and around the fillet radius is increased further together with the number of elements along the perimeter of the holes. The rivet mesh corresponding to mesh 1 and mesh 2 is the same, whereas, for mesh 3, the number of elements along the rivet shank and around its perimeter is increased.

The fatigue damage calculated for a single train passage (BS 5400 train No 7) for the three different mesh densities shown in Figure 3 is shown in Figure 4. The results, which are presented for different locations within the different components of the connection, are presented for the case of having a $100 \mathrm{MPa}$ clamping stress in the rivets. Moreover, the results shown in Figure 4 are, at each location, related to the most damaging notch tips.

Comparison of the fatigue damage associated with meshes 1 and 2 reveals considerable differences, which, in some cases, may be as high as 50\%. By increasing the mesh density further (mesh 3), the differences in damage are significantly reduced with a maximum difference of $15 \%$ between meshes 2 and 3. Figure 4 demonstrates that the TCD volume method results in mesh convergence in all the connection components which are associated with different types of notches (i.e. holes, fillets, corners). 

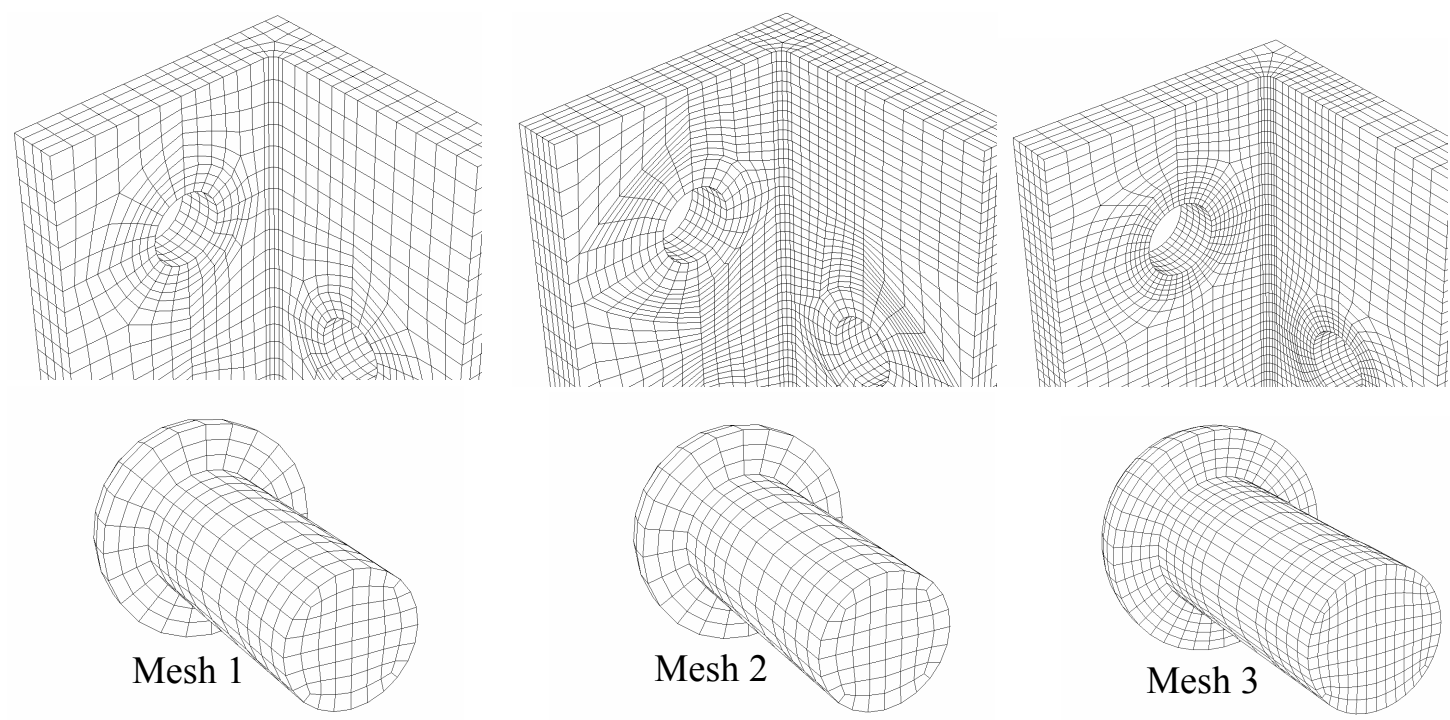

Figure 3. Parts of the FE model pertaining to three different mesh densities.

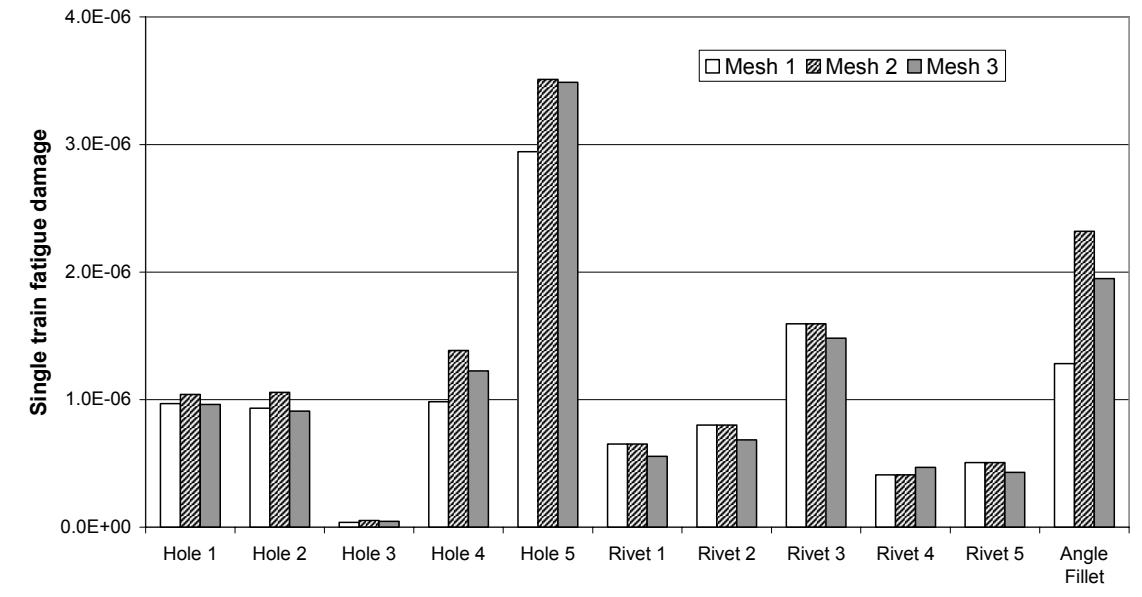

Figure 4. Fatigue damage at different locations of the connection for three different mesh densities (BS 5400 train No 7, rivet clamping stress $=100 \mathrm{MPa}$ ).

\subsection{Fatigue damage}

The total fatigue damage of the different connection components over the period 1970 to 2007 is calculated using the combined stress histories of all four trains of the BS 5400 medium traffic. The fatigue damage estimates obtained from the refined (global-local) model shown in Figure 1 coupled with the TCD $\left(D_{l}\right)$ are compared with the estimates obtained in a previous investigation by the authors (Imam et al. 2006). The latter damage estimates $\left(D_{g}\right)$ were obtained through a crude FE bridge model using nominal stress histories coupled with the class pertaining to wrought iron riveted details proposed by Network Rail (Railtrack 2001). The results are presented in Figure 5 in terms of the ratio $D_{l} / D_{g}$ as well as the remaining fatigue lives, assuming no load evolution in the future. It should be noted that, in the case of the crude model, a single fatigue damage estimate is obtained for the entire connection. The reason is that damage calculations are carried out with the use of a generic, detail-specific S-N curve.

It can be seen in Figure 5 that, for the critical regions of the connection (hole 5 , rivet 3 and angle fillet), the use of the TCD results in higher damage estimates. The differences between the predictions of the two models, for the critical regions, are lower in the case of the higher rivet 
clamping stress of $200 \mathrm{MPa}$. Depending on the clamping stress, Figure 5 shows that the traditional assessment method (Imam et al. 2006) can underestimate fatigue damage by a factor of 3.5. Furthermore, the remaining fatigue life estimates demonstrate the expected beneficial effect of the clamping stress.

The results in Figure 5 indicate that hole 5 on the stringer part of the connection (see Fig. 1b) is the most highly damaged part of the connection irrespective of the level of clamping. Furthermore, as a result of out-of-plane deformations, rivet 3 on the cross-girder part of the connection (see Fig. 1b) appear to be the next most highly damage component of the connection for the case of $100 \mathrm{MPa}$ clamping stress. However, the results presented in Figure 5 should be viewed within the uncertainty context associated with manufacturing (clamping stress) and material properties (fatigue limit, stress intensity threshold, S-N curves).

One of the most important advantages of the TCD is that it can be applied for the fatigue assessment of any type of notch geometry. It eliminates the need of classifying the detail to be investigated according to the various classifications proposed by the bridge standards (BS 5400 1980, AASHTO 1998). This is due to the fact that only the plain (un-notched) material properties are required for the application of this method (i.e. the fatigue limit and the threshold stress intensity factor), both of which can be determined in a straightforward way through fatigue testing. As a result, within the context of the TCD, there is no need of extensive testing of different connection details.

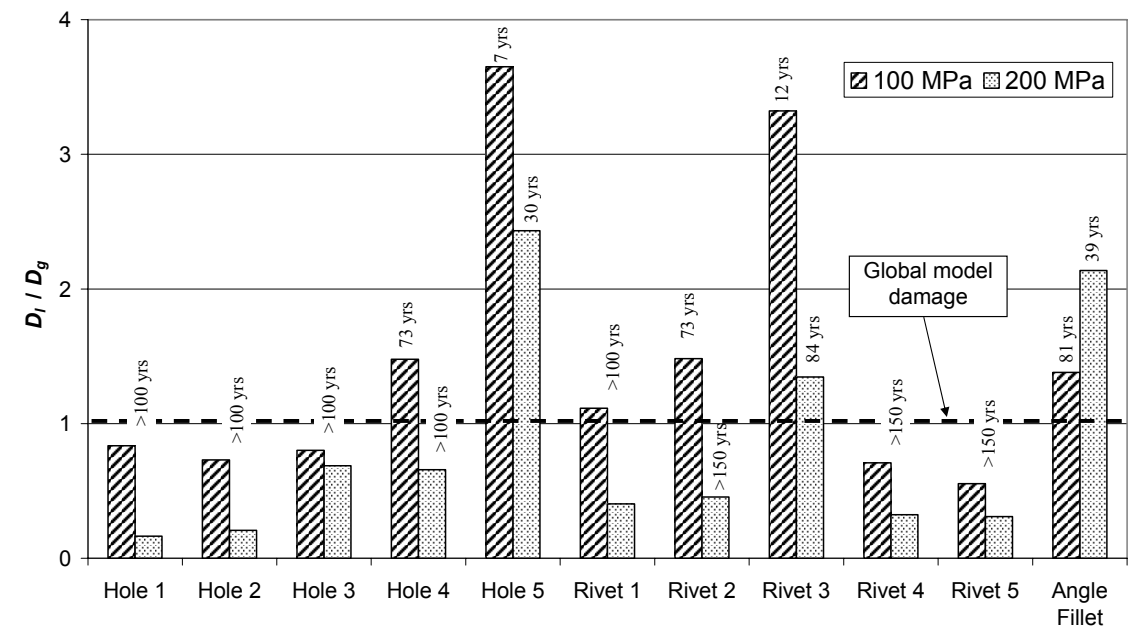

Figure 5. Comparison of total fatigue damage between crude (Imam et al. 2006) and global-local (TCD) models for the period 1970-2007 and corresponding remaining fatigue lives.

\section{CONCLUSIONS}

This paper has presented fatigue damage estimates for a typical, short-span, riveted railway bridge. These were obtained through a novel fatigue assessment approach which employs the Theory of Critical Distances (TCD). The TCD, rather than relying on nominal stresses or the stress at the notch, considers the entire stress field ahead of the notch. This study has demonstrated that, with FE mesh refinement, reasonable convergence in fatigue damage estimates is achieved through the use of the TCD. This contrasts the case of considering notch stresses where fatigue damage, with increasing mesh density, becomes unbounded.

The total damage of the different connection components was estimated using a combined load history resulting from the passage of trains over the bridge. The damage estimates were compared with the estimates calculated using the traditional approach i.e. nominal stresses coupled with the detail-specific S-N curve. The results have shown that the traditional approach tends to underestimate the fatigue damage of the critical regions of the connection, by a maxi- 
mum factor of 3.5 in the case of low rivet clamping force. These differences for the critical regions were found to decrease for higher clamping force.

Although the TCD requires considerable post-processing, especially for three-dimensional bridge applications, its reliance on straightforward material characterisation and its convergent characteristics make it appealing for the fatigue assessment of complex bridge details, such as riveted stringer-to-cross-girder connections. Once the application of this method is validated for bridges, refined fatigue assessment will be possible without the need for extensive testing of different connection details.

\section{REFERENCES}

Abouelmaaty, W., Maragakis, E., Itani, A., Douglas, B. 1999. Fatigue testing of double-angle connections of steel railroad bridges. Transportation Research Record 1688: 46-52. Transportation Research Board, Washington D.C.

Al-Emrani, M. 2002. Fatigue in riveted railway bridges - A study of the fatigue performance of riveted stringers and stringer-to-floor-beam connections. PhD Thesis. Chalmers University of Technology, Sweden.

Al-Emrani, M. 2005. Fatigue performance of stringer-to-floor-beam connections in riveted railway bridges. Journal of Bridge Engineering (ASCE) 10(2): 179-185.

Araujo, J.A., Susmel, L., Taylor, D., Ferro, J.C.T., Mamiya, E.N. 2007. On the use of the theory of critical distances and the modified Wohler curve method to estimate fretting fatigue strength of cylindrical contacts. International Journal of Fatigue 29(1): 95-107.

American Association of State Highway Transportation Officials (AASHTO). 1998. LRFD bridge design specifications. $2^{\text {nd }}$ ed., Washington, D. C.

Bellett, D., Taylor, D., Marco, S., Mazzeo, E., Guillois, J., Pircher, T. 2005. The fatigue behaviour of three-dimensional stress concentrations. International Journal of Fatigue 27(3): 207-21.

BS 5400. 1980. Steel, concrete and composite bridges: Part 10: Code of practice for fatigue. London: British Standards Institute.

Imam, B., Righiniotis, T.D., Chryssanthopoulos, M.K., Bell, B. 2006. Analytical fatigue assessment of a typical riveted UK rail bridge. Proceedings of the Institution of Civil Engineers (ICE) - Bridge Engineering 159(3): 105-16.

Imam, B.M., Righiniotis, T.D., Chryssanthopoulos, M.K. 2007. Numerical modelling of riveted railway bridge connections for fatigue evaluation. Engineering Structures 29(11): 3071-3081.

Railtrack. 2001. RT/CE/C/025, Railtrack line code of practice: The structural assessment of underbridges, Railtrack.

Susmel, L. 2004. A unifying approach to estimate the high-cycle fatigue strength of notched components subjected to both uniaxial and multiaxial cyclic loadings. Fatigue and Fracture of Engineering Materials and Structures 27(5): 391-411.

Susmel, L. \& Taylor, D. 2003. Fatigue design in the presence of stress concentrations. Journal of Strain Analysis and Engineering Design 38(5): 443-52.

Taylor, D. 1999. Geometrical effects in fatigue: a unifying theoretical model. International Journal of Fatigue 21(5): 413-20.

Taylor, D. \& Wang, G. 2000. The validation of some methods of notch fatigue analysis. Fatigue and Fracture of Engineering Materials and Structures 23(5): 387-94.

Taylor, D., Bologna, P., Bel Knani, K. 2000. Prediction of fatigue failure location on a component using a critical distance method. International Journal of Fatigue 22(9): 735-42.

Taylor, D., Barrett, N., Lucano, G. 2002. Some new methods for predicting fatigue in welded joints. International Journal of Fatigue 24(5): 509-18. 